\title{
Correction to: Game Theoretic Approaches to Mitigate Cloud Security Risks: An Initial Insight
}

Abdelkarim Ait Temghart, M'hamed Outanoute, and Mbarek Marwan

\section{Correction to:}

Chapter "Game Theoretic Approaches to Mitigate Cloud

Security Risks: An Initial Insight" in: M. Fakir et al. (Eds.):

Business Intelligence, LNBIP 416,

https://doi.org/10.1007/978-3-030-76508-8_24

In the originally published version of chapter 24, the author Driss Ait Omar was erroneously included in the author list. This has now been corrected. 